\title{
Clinical Evaluation of Tachosil@ in Hemostasis and Aerostasis in Abdominal and Thoracic Operations
}

\author{
Mohammad Vaziri ${ }^{1}$, Fatemeh Jesmi ${ }^{2}$, Mohadesseh Pishgahroudsari ${ }^{3}$ and Taiebeh Mohammadi Farsani*4 \\ ${ }^{1}$ MD, Associate Professor of Thoracic surgery, Minimally Invasive Surgery Research Center, Iran University of Medical Sciences, Tehran, Iran, vaziri.m@iums. \\ ac.ir, dr.mohammad.vaziri1340@gmail.com
}

${ }^{2}$ Research Fellow, Minimally Invasive Surgery Research Center, Iran University of Medical Sciences, Tehran, Iran, fatemehjesmi@yahoo.com

${ }^{3}$ BS of Statistics, Minimally Invasive Surgery Research Center, Iran University of Medical Sciences, Tehran, Iran, pishgah.m@kar.iums.ac.ir

${ }^{4} \mathrm{PhD}$, Minimally Invasive Surgery Research Center, Iran University of Medical Sciences, Tehran, Iran, Department of Medical Biotechnology, Isfahan (Khorasgan) Branch, Islamic Azad University, Isfahan, Iran, taiebeh.mohammadi@khuisf.ac.ir

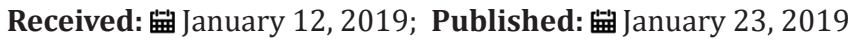

*Corresponding author: Taiebeh Mohammadi Farsani, Minimally Invasive Surgery Research Center, Iran University of Medical Sciences, Tehran, Iran, Department of Medical Biotechnology, Isfahan (Khorasgan) Branch, Islamic Azad University, Isfahan, Iran

Abstract

Background: Prolonged bleeding during abdominal surgery and persistent air leakage following thoracic operations present a risk for the patient and increase the time and cost of surgery. These are considered major limiting factors for discharge from hospital.

Object: The aim of this study was to evaluate the sealing capacity of Tachosil $®$ for hemorrhage control and the closure of alveolar air leaks, respectively.

Methods: A total of 30 patients undergoing general abdominal and thoracic surgeries were enrolled in a single-center study to evaluate the efficacy of Tachosil@. After the application of trial treatment, hemostasis in abdominal surgeries was evaluated at 3 min and cessation of air leak in thoracic operations was intraoperatively evaluated by submersion of the resection site in saline (primary end point). Post-operative bleeding or air leak was also demonstrated (secondary end point).

Results: Thirty patients including 18 men and 12 women with a mean age of 38 (age range 14-73 years) underwent thirteen different types of surgeries such as thoracotomy, cholecystectomy, splenectomy, nephrectomy and hepatic repair. During abdominal surgeries the time needed to achieve hemostasis was about 2.5 minutes and minor postoperative bleeding was noted in two patients (6.6\%) undergoing acute cholecyctitis and liver trauma repair who were not required additional intervention. In thoracic operations, air leak control was obtained in all patients, but postoperative minor air leak was observed in 7 patients (36\%) which disappeared on the third day with no required intervention and therefore is not considered a surgical complication. Thus, bleeding and air leak control failure rates in this study were zero and surgical site infection or any other complication attributed to Tachosil@ was not observed.

Conclusion: Tachosil $®$ is a safe and well tolerated material in obtaining effective and fast intra-operative hemostasis and air leak control in abdominal and thoracic surgical procedures respectively.

Keywords: Hemostasis; Air Leak; Surgical Adhesive

\section{Introduction}

Traditionally, methods such as suturing, cauterization or Argon beam laser were used in order to control non-active bleeding in hepatic and splenic injuries which due to fragility of these tissues, may lead to further laceration and bleeding [1,2]. On the other hand, Argon beam laser is not available in the most hospital settings and in lung tissue, due to its distinct structure, The use of sutures is quite problematic and frequently results in lung tearing, especially in underlying parenchymal diseases such as emphysema. In addition, cauterization has no place in lung surgery in this regard [1].
The use of a surgical product that can cause rapid hemostasis has the following advantages: availability, ease of use in tissues which are easily ruptured such as spleen, pliability (to be tailored to the preferences of the relevant surfaces), usability in both open and laparoscopic surgery, good adhesiveness in the presence of body fluids, blood, absorption into the body without leaving a side effect and ability to be maintained in the room temperature without refrigeration [2]. Many products with different approaches and targets like collagen, gelatin are available on the market [2,3]. One of the most recent developments are fibrin-based haemostats such 
as Tachosil@ [3]. Tachosil@ surgical patch is the only product with the above-mentioned characteristics that contains a combination of collagen, fibrinogen and human thrombin. It has the ability to be used in a wide variety of open and laparoscopic surgical procedures and various tissues such as the spleen, liver, kidney and lung $[4,5]$.

Additional significant application of Tachosil@ includes preventing air leak in various thoracic operations [6]. The efficacy and safety of Tachosil $®$ for preventing air leaks after pulmonary lobectomy has been reported in several trials $[7,8]$. Regarding the high burden of traumatic injuries as a national problem and taking into consideration the large number of its related surgeries, overall benefit/risk ratio of using a product to accelerate the creation of hemostasis and reduce the operative time, is quite high. Accordingly, we designed a research project to investigate the clinical benefits and outcomes of such a product and in the meanwhile, to teach and make surgical residents become familiar with a new method.

\section{Methods}

In this trial, 30 patients (18 men and 12 women, $14-73$ years old and mean age of 38) were studied. They underwent thoracic and abdominal surgeries in Rasoul Akram General Hospital, Tehran, Iran, during a three-month period from June till September 2012 . This study were done by application of Tachosil $₫$ (Ferric Chemical Co.Tehran, Iran) in prevention and treatment of bleeding and air leak during abdominal surgeries (such as hepatic, splenic and renal operations) and thoracic surgeries including lung decortication and traumatic lung repair.

Tachosil@ is a medical sponge containing $5.5 \mathrm{mg}$ of fibrinogen and two unit's thrombin per $\mathrm{cm} 2$ in which the active area is marked with yellow. The size used in the majority of surgical procedures including laparoscopic operations is $4.8 * 4.8 \mathrm{~cm}$. This product, like other sealants used, undergoes denaturation when exposed to alcohol, iodine or heavy metals (antiseptic solution), so contact with povidone iodine solution is to be avoided. This product is stored at room temperature (less than 25 Celsius degree) and its shelf life is 36 months. When using this product, the wound was cleaned so that it contained no blood or other fluid, and Tachosil@, after getting wet, was immediately used. The yellow side was put on the wound and pressed for 3 minutes with a wet gas after which the wet gas was removed and Tachosil $®$ with a super adhesive strength remained in the organ or surgical scars.

In thoracic surgeries, air leak control is achieved during surgery by increasing the air way pressure to $30 \mathrm{~cm} \mathrm{H} 2 \mathrm{O}$ and putting the repaired lung under a sterile solution to observe the possible bubbling from pulmonary parenchyma, which requires additional treatment. In order to identify whether patients with postoperative air leak need any invasive or surgical intervention, this observation was classified as minor (bubbling only following cough i.e., increased airway pressure), moderate (streamline bubbling) and severe (continuous bubbling with normal breathing). Moderate and severe air leaks were considered a surgical complication that frequently need re-operation.

\section{Inclusion Criteria}

The following procedures was included in the efficacy assessment study of the Tachosil@: laparotomy for abdominal trauma with grade I or II splenic and hepatic injuries, partial splenectomy, resection of a liver mass such as metastasis, open and laparoscopic cholecystectomy, renal trauma, surgical resection of a superficial renal mass, thoracotomy for traumatic lung lacerations, air leak control in lung decortication and wedge resection.

\section{Exclusion Criteria}

Neurological surgery, vascular surgery, gastrointestinal anastomosis, hemodynamically unstable patient, damage to the spleen, liver and kidneys more than grade II-pregnant and lactating women who are undergoing surgery. The resulting information was recorded in an appropriately designed questionnaire.

Statistical Analysis: Frequency (percentage) of the patients in each group is reported if relevant. In addition, quantitative variables are described using mean \pm Standard Deviation (SD).

\section{Results}

In this study 30 patients undergoing thoracic and abdominal surgeries, were assessed using Tachosil@. The patients included 18 men (60\%) and 12 women (40\%), with age range of $14-73$ and mean age of 38. The types of surgeries included 13 various operations including thoracotomy (for 9 different diseases), cholecystectomy, splenectomy, nephrectomy, and liver laceration repair. The number of surgeries included 19 cases of thoracotomy, including 8 cases of decortication, 3 cases of spontaneous pneumothorax repair, 2 cases of pneumonectomy, 1 case of lobectomy, 1 case of bullae resection, 1 case of mediastinal tumor resection, 1 case of wedge resection in diffuse lung disease, 1 case of pulmonary laceration repair due to gunshot, and 1 hydatid cyst surgery. Laparoscopic cholecystectomy was performed in 6 cases, among which 1 was acute cholecystitis. Splenectomy was performed in 3 cases, 2 of which were laparoscopically done. There were also 1 case of nephrectomy and 1 liver laceration repair.

Postoperative infection rate was zero and no patient was complicated with surgical site infection. The mean time needed for hemostasis was 2.5 minutes (range from 1.5 to 3 minutes). Postoperative bleeding occurred in 2 patients (in liver laceration repair and cholecystectomy due to acute cholecystitis), which was $6.6 \%$ of all patients. Considering only the abdominal surgeries this would be $18 \%$. All cases of postoperative bleedings ceased spontaneously with no invasive or surgical intervention on second or third postoperative day, i.e. failure rate of zero (Table 1). 
Table 1: Result of using Tachosil® in 11 cases of abdominal surgery.

\begin{tabular}{|c|c|c|c|}
\hline Surgery type & Number of Tachosil@s used & $\begin{array}{c}\text { Mean intra-operative bleeding } \\
\text { time (min) }\end{array}$ & $\begin{array}{c}\text { Number of cases with } \\
\text { Postoperative bleeding }\end{array}$ \\
\hline Laparoscopic Cholecystectomy & 6 & 2 & 1 \\
\hline Splenectomy & 3 & 1.5 & 0 \\
\hline Nephrectomy & 1 & 2 & 0 \\
\hline Liver laceration repair & 1 & 3 & 1 \\
\hline
\end{tabular}

In thoracotomies 16 out of 19 patients required Tachosil@ for air leak control, which included decortication, bullae resection, lobectomy, spontaneous pneumothorax repair, wedge resection in diffuse pulmonary disease, pulmonary repair due to gunshot, and hydatid cyst resection. postoperative minor air leak occurred in seven (36\%) cases including decortication, spontaneous pneumothorax repair, wedge resection, and pulmonary laceration repair due to gunshot (Table 2). Spontaneous cessation of minor air leaks, which are not considered as complication, was observed in all patients. Thus, failure rate of air leak control was zero.

Table 2: Results of using Tachosil® in 19 cases of thoracotomy.

\begin{tabular}{|c|c|c|c|c|}
\hline Type of surgery & $\begin{array}{l}\text { Number of Tachosil }{ }^{\circledR} \\
\text { used during operation }\end{array}$ & $\begin{array}{l}\text { Air leak control during } \\
\text { operation }\end{array}$ & $\begin{array}{l}\text { Air leak after the } \\
\text { operation }\end{array}$ & $\begin{array}{l}\text { Air leak control after the } \\
\text { operation without any } \\
\text { complication }\end{array}$ \\
\hline $\begin{array}{l}\text { Resection of Pulmonary } \\
\text { bullae }\end{array}$ & 1 & + & One case & + \\
\hline Decortication & 8 & + & Three Cases & + \\
\hline Pneumonectomy & 2 & + & -- & + \\
\hline Lobectomy & 1 & + & -- & + \\
\hline $\begin{array}{c}\text { Spontaneous Pneumothorax } \\
\text { repair }\end{array}$ & 3 & + & One case & + \\
\hline $\begin{array}{l}\text { Mediastinal tumor } \\
\text { resection }\end{array}$ & 1 & + & -- & + \\
\hline $\begin{array}{l}\text { Resection in Wedge Diffuse } \\
\text { Lung Disease }\end{array}$ & 1 & + & One case & + \\
\hline $\begin{array}{l}\text { Pulmonary laceration repair } \\
\text { due to gunshot }\end{array}$ & 1 & + & One case & + \\
\hline Pulmonary Hydatid cyst & 1 & + & -- & + \\
\hline
\end{tabular}

\section{Discussion}

The success of any surgical procedure is based on adequate hemostasis because ineffective local hemostasis is the major cause of bleeding during surgery. Many different biomaterial products can be used to achieve the same goal with less tissue injury and reduced cost and in a more rapid and easier manner $[9,10]$. Tachosil $囚$, a collagen patch coated with human fibrinogen and thrombin, is used to improve postsurgical haemostasis $[9,11,12]$. In our study, during abdominal surgeries the time needed to achieve hemostasis was about 2.5 minutes and postoperative minor bleeding was noted in two patients $(6.6 \%)$ undergoing acute cholecyctitis and liver trauma repair who did not required additional intervention.

Kawasaki showed that Tachosil@ provided effective homeostasis within 5 min of application in 64 patients [5]. Their results of studies showed Tachosil@ has noninferior hemostatic efficacy compared with TachoComb, and a similar safety profile, in patients undergoing liver resection. Their results therefore suggested that the beneficial effects of Tachosil ${ }^{\circledR}$ are similar to those of TachoComb and that Tachosil® is an effective mean of providing hemostasis in Japanese patients during surgery [5]. The clinical efficacy of Tachosil@ was shown by a clinical study in hepatic surgery [13]. In this the study, Tachosil@ were proved to be better than argon beamer in obtaining effective and fast intraoperative hemostasis [13]. In the field of hepatic surgery, a trial demonstrated the efficacy and safety of Tachosil ${ }^{\circledR}$ versus argon beamer in liver resection [14]. In the two groups of patients, the mean hemostasis time was less when Tachosil@ was used (3-6 minutes) compared with argon beamer (5.0 minutes $\mathrm{P}=0.008)$. Postoperative drainage volume, drainage fluid, and drainage duration did not differ between the two groups. The study confirmed that Tachosil@ hemostasis is significantly faster than argon beamer after liver resection.

Briceño in a clinical trial, used Tachosil $®$ in which 115 patients. These patients were distributed into two groups for major and minor hepatectomies, with or without application of a carrierbound collagen sponge on the raw surface of the liver. Their results showed that Tachosil ${ }^{\circ}$ was effective in decreasing drainage volume, postoperative blood transfusion, complications and hospital stay [15]. Grottke investigated the efficacy of Tachosil ${ }^{\circledR}$ in stopping severe bleeding in a coagulopathic pig model with blunt liver injury $[16,17]$. The study analyzed two groups of pigs that were previously prepared by splenectomy and cystotomy and 
by inducing coagulopathy. Subsequently, a grade III liver injury was induced. At this point, animals were randomly assigned to receive a placebo patch or Tachosil $囚$. Coagulation parameters and hemodynamic variables as well as the chosen treatment were monitored for 2 hours after injury and patch placement. All animals treated with Tachosil@ survived, whereas $100 \%$ of the control group died before reaching the end of the observation period $(\mathrm{P}<$ 0.001).

Additional significant application of Tachosil@ includes preventing air leak in various thoracic operations. In thoracic operations, air leak control was obtained in all patients, but postoperative minor air leak was observed in 7 patients (36\%) which disappeared on the third day with no intervention required and therefore is not considered a surgical complication. In this study, bleeding and air leak control failure rates were zero and surgical site infection or any other complication attributed to Tachosil@ was not observed. Air leaks have been reported to occur in 48-88\% of patients undergoing lung resection [6].

Benefits of Tachosil@ application for splenic trauma or fragile or atraumatic damaged spleen have been reported. In hematologic diseases, Tachosil ${ }^{\circledR}$ may be useful in clotting and for blood composition disorders. In many fields of abdominal surgery such as pancreatic and splenic procedures, fragile tissue is a major concern and use of this sealant is effective in hemostasis and also prevents leakage. In fact, pancreatic surgery is one of the most difficult surgical fields due to the risk of pancreatic fistula. We had no case of pancreatic lesion to be included in our study but the results of Tachosil@ use in three cases of splenic resections, including two laparoscopic splenectomies, were quite encouraging and helpful. In our study Tachosil ${ }^{\circledR}$ was used in six cases of laparoscopic cholecystectomy and two cases of laparoscopic splenectomy with no adverse or undesirable outcome.

Our study also demonstrated the efficacy of Tachosil $®$ in reducing postoperative air leaks in patients undergoing various thoracic operations, especially in a group of high-risk patients in whom intraoperative and/or postoperative air leaks are expected. It is to be noted that our study presents some limitations. The number of patients was limited by the nature of the study itself, the aim of which was to enroll diverse surgical patients in whom a clear indication to use Tachosil@ could be demonstrated. In addition, this was intended as a pilot study with no formal calculation of the sample size needed to achieve a statistical power for confident evaluation of our results.

\section{Conclusion}

Tachosil ${ }^{\circledR}$ is a fast, safe and effective modality to achieve hemostasis and aerostasis in various abdominal and thoracic operations with no imposed tissue injury which eliminates the use of invasive suturing techniques in delicate tissues.

\section{Acknowledgement}

We gratefully appreciate patients and their families for their cooperation in this manuscript.

\section{References}

1. Seyednejad H, Imani M, Jamieson T, Seifalian AM (2008) Topical haemostatic agents. Br J Surg 95(10): 1197-1225.

2. Vecchio R, Catalano R, Basile F, Spataro C, Caputo M, et al. (2016) Topical hemostasis in laparoscopic surgery. G Chir 37(6): 266-270.

3. Berrevoet F, de Hemptinne B (2007) Clinical application of topical sealants in liver surgery: Does it work? Acta Chir Belg 107(5): 504-507.

4. Kawasaki S, Origasa H, Tetens V, Kobayashi M (2017) Comparison of TachoSil and TachoComb in patients undergoing liver resection-A randomized, double-blind, non-inferiority trial. Langenbecks Arch Surg 402(4): 591-598.

5. Varela G, Jiménez MF, Novoa N, Aranda JL (2005) Estimating hospital costs attributable to prolonged air leak in pulmonary lobectomy. Eur J Cardiothorac Surg 27(2): 329-333.

6. Marta GM, Facciolo F, Ladegaard L, Dienemann H, Csekeo A, et al. (2010) Efficacy and safety of TachoSil® versus standard treatment of air leakage after pulmonary lobectomy. Eur J Cardiothorac Surg 38(6): 683-689.

7. Anegg U, Lindenmann J, Matzi V, Smolle J, Maier A, et al. (2007) Efficiency of fleece-bound sealing (TachoSilß) of air leaks in lung surgery: A prospective randomised trial. Eur J Cardiothorac Surg 31(2): 198-202.

8. Stellingwerff M, Brandsma A, Lisman T, Porte RJ, (2012) Prohemostatic interventions in liver surgery. Semin Thromb Hemost 38(3): 244-249.

9. Ekberg H, Tranberg K, Andersson R, Jeppsson B, Bengmark S (1986) Major liver resection: Perioperative course and management. Surgery $100(1): 1-8$.

10. Hüttner FJ, Mihaljevic AL, Hackert T, Ulrich A, Büchler MW, et al. (2016) Effectiveness of Tachosil $®$ in the prevention of postoperative pancreatic fistula after distal pancreatectomy: A systematic review and metaanalysis Langenbecks Arch Surg 401(2): 151-159.

11. Frilling A, Stavrou GA, Mischinger HJ, de Hemptinne B, Rokkjaer M, et al. (2005) Effectiveness of a new carrier-bound fibrin sealant versus argon beamer as haemostatic agent during liver resection: A randomised prospective trial. Langenbecks Arch Surg 390(2): 114-120.

12. Fischer L, Seiler CM, Broelsch CE, de Hemptinne B, Klempnauer J, et al. (2011) Hemostatic efficacy of TachoSil in liver resection compared with argon beam coagulator treatment: An open, randomized, prospective, multicenter, parallel-group trial. Surgery 149(1): 48-55.

13. Briceño J, Naranjo A, Ciria R, Díaz-Nieto R, Sánchez-Hidalgo JM, et al. A prospective study of the efficacy of clinical application of a new carrierbound fibrin sealant after liver resection. Arch Surg 145(5) :482-488.

14. Grottke O, Braunschweig T, Daheim N, Coburn M, Grieb G, et al. Effect of TachoSil in a coagulopathic pig model with blunt liver injuries. J Surg Res 171(1): 234-239.

15. Carbon RT, Baar S, Waldschmidt J, Huemmer HP, Simon SI (2002) Innovative minimally invasive pediatric surgery is of therapeutic value for splenic injury. J Pediatr Surg 37(8): 1146-1150.

16. Schwaitzberg SD, Chan MW, Cole DJ, Read M, Nichols T, et al. (2004) Comparison of poly-N-acetyl glucosamine with commercially available topical hemostats for achieving hemostasis in coagulopathic models of splenic hemorrhage. J Trauma 57(1): S29-S32.

17. Tagliabue F, D’Angelo C, Zuccon W, Giorgetta C, Gambarini F, et al. (2007) Use of Tachosil in splenectomy in patients with clotting and blood composition disorders. Minerva Chir 62(1): 73-78. 
ISSN: 2574-1241

DOI: $10.26717 / B J S T R .2019 .13 .002412$

Taiebeh Mohammadi Farsani. Biomed J Sci \& Tech Res

(C) (i) This work is licensed under Creative

Submission Link: https://biomedres.us/submit-manuscript.php

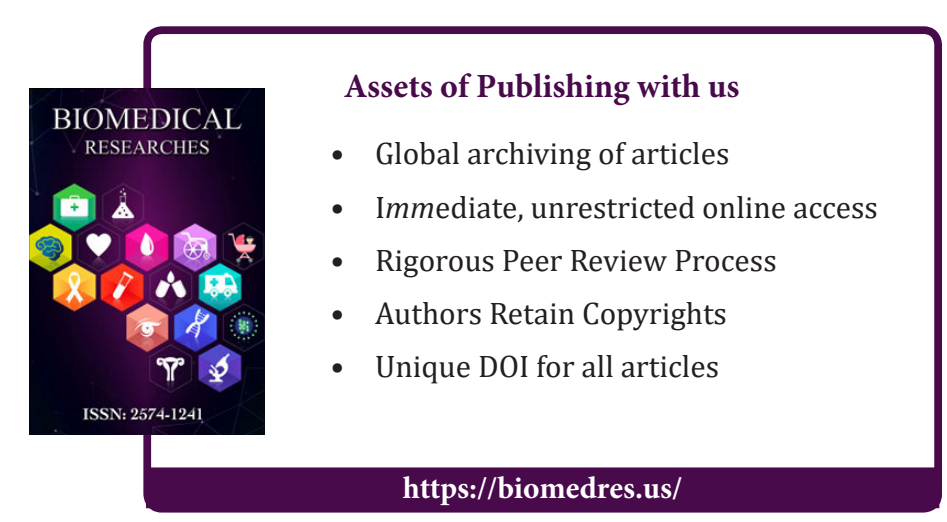

